\section{Response to: 'Risk of severe infection following rituximab and the efficacy of antimicrobial prophylaxis' by Wallace et al}

We thank Dr Wallace et al for their response to our recently published article 'Trimethoprim-sulfamethoxazole prophylaxis prevents severe/life-threatening infections following rituximab in antineutrophil cytoplasm antibody-associated vasculitis', highlighting some methodological limitations of our study. ${ }^{12}$

One of the limitations mentioned by Wallace and colleagues is the inclusion of incident and prevalent cases and since only 15 out of 192 patients were incident cases, generalisability of our findings for this subset of patients may not be possible. We agree that the use of immunosuppression prior to initiation of rituximab likely confers a risk to develop infectious complications after rituximab administration. Cyclophosphamide was used to control disease in 62 patients the year before rituximab was initiated. Among these, 53 patients had no severe infection (median cyclophosphamide exposure $7 \mathrm{~g}$, range $0.66-45 \mathrm{~g}$ ), while 9 patients receiving cyclophosphamide the index year before had a severe infection following rituximab (median cyclophosphamide exposure $4.8 \mathrm{~g}$, range $0.8-10 \mathrm{~g}$ ). While this argues against an immediate impact of cyclophosphamide before rituximab on the risk of severe infections (53/143 with no severe infection against 9/49 with infection received cyclophosphamide), we also believe there exists a carry-over effect of diverse immunosuppressive measures on the risk to develop infectious complications. A recent analysis of non-antineutrophil cytoplasm antibody (ANCA)-associated vasculitis cases using The Health Improvement Network found that glucocorticoid exposure confers a high risk for lower respiratory tract infections. ${ }^{3}$ Since a majority of infections observed in our analysis were related to the lower respiratory tract (63/95), the 'true influence' of concomitant glucocorticoid treatment may have been underestimated in our study. Moreover, we did not calculate the damage attributable to active vasculitis in this study. It is likely that patients with a higher Vasculitis Damage Index score are in particular prone to infectious complications.

We acknowledged the fact that prescription of trimethoprimsulfamethoxazole (TMP-SMX) was time-varying, which means that if patients stopped TMP-SMX and developed their first severe infection afterwards, they were assigned to the unexposed group. Since analyses were performed with first infectious complication as the primary outcome, no such adjustments were undertaken for subsequent infectious complications.

Confounding by indication or contraindication is a limitation of observational studies. Novel approaches such as activecomparator design and new-user design could be helpful to mitigate such biases. ${ }^{4}$ We did not perform an active-comparator study, which would comprise a cohort of patients receiving cyclophosphamide as part of their induction treatment. Moreover, a new-user study was not feasible since recently diagnosed or relapsing patients were unlikely to receive a cyclophosphamidebased induction treatment after results of the Rituximab in ANCA-Associated Vasculitis (RAVE) trial have been published. ${ }^{5}$ As stated by Wallace et al, there were differences in the prescription of TMP-SMX in our study. Patients with ear, nose and throat (ENT) involvement were more likely to receive TMPSMX prophylaxis (online supplementary table S5). ${ }^{2}$ We agree that patients with predominant ENT involvement are less likely to receive 'intensive' immunosuppressive therapy; however, our patients with TMP-SMX prescription had a non-significantly higher BVAS $(p=0.093)$ and similar rates of kidney and lung involvement. Patients receiving TMP-SMX prophylaxis had lower CD4 T-cell count and were exposed to more cyclophosphamide the year before rituximab and had a higher concomitant glucocorticoid exposure (online supplementary table S5). ${ }^{2}$ Thus, we conclude that the subset of patients receiving prophylaxis in our study had at least a comparable disease severity compared with those not receiving TMP-SMX.

Despite its proven efficacy to reduce the occurrence of infectious complications, TMP-SMX exhibits a variety of severe and non-severe side effects. The study by Stegeman and colleagues used a therapeutic dosage of TMP-SMX (twice daily, 800/160 $\mathrm{mg}$ ) for a period of 2 years and found side effects in 8/41 patients. ${ }^{6}$ Of note, only a minority of patients received a similar high dose of TMP-SMX in our cohort, while prophylaxis consisted of 480 mg or 960 mg every other day in most cases (online supplementary table S4). ${ }^{2}$ TMP-SMX was stopped in 5 out of 73 patients in our cohort due to adverse events. ${ }^{2}$ A recent study investigated the role of TMP-SMX in patients with prolonged exposure to high-dose glucocorticoids and found a reduction in the occurrence of infections with Pneumocystis jirovecii. Among 262 patients receiving prophylaxis, two cases with serious adverse events attributable to TMP-SMX were reported (pancytopenia and Stevens-Johnson syndrome), while mild side effects were found in another 34 cases. $^{7}$ A recent randomised controlled trial tested the role of TMP-SMX (800/160 mg twice daily for 1 week) against placebo for the management of uncomplicated skin abscess. Among 630 subjects receiving TMP-SMX, only one case with pancytopenia was noted and in general, no difference in the frequency of side effects was reported. ${ }^{8}$ No such investigations including cases with ANCA-associated vasculitis have been performed so far, but larger studies in other entities and as an anti-infective measure point towards an acceptable safety profile of TMP-SMX.

In clinical practice, we sometimes see patients with severe side effects attributable to TMP-SMX prophylaxis. There is a need to balance risks and benefits of such prophylaxis, but in the absence of prospective studies (observational or ideally a randomised controlled trial), we recommend prophylaxis in those patients with comorbidities or receiving background immunosuppression (mainly glucocorticoids) or with an exposure to a variety of immunosuppressive agents in the past.

\section{Andreas Kronbichler $\odot$, ${ }^{1,2}$ Julia Kerschbaum ${ }^{2}$ \\ ${ }^{1}$ Vasculitis and Lupus Clinic, Addenbrooke's Hospital, Cambridge, UK ${ }^{2}$ Department of Internal Medicine IV (Nephrology and Hypertension), Anichstraße, Innsbruck, Austria}

Correspondence to Dr Andreas Kronbichler, Department of Internal Medicine IV (Nephrology and Hypertension), Anichstraße, Innsbruck 6020, Austria; andreas.kronbichler@i-med.ac.at

Handling editor Josef S Smolen

Competing interests None declared.

Patient consent for publication Not required.

Provenance and peer review Commissioned; internally peer reviewed.

(c) Author(s) (or their employer(s)) 2020. No commercial re-use. See rights and permissions. Published by BMJ.

Check for updates

To cite Kronbichler A, Kerschbaum J. Ann Rheum Dis 2020;79:e41.

Received 10 January 2019

Accepted 11 January 2019

Published Online First 30 January 2019 


\section{Linked}

http://dx.doi.org/10.1136/annrheumdis-2019-215009

Ann Rheum Dis 2020;79:e41. doi:10.1136/annrheumdis-2019-215036

ORCID iD

Andreas Kronbichler http://orcid.org/0000-0002-2945-2946

\section{REFERENCES}

1 Wallace Z, Hyon C, Stone J. Risk of severe infection following rituximab and the efficacy of antimicrobial prophylaxis. Ann Rheum Dis 2020;79:e40.

2 Kronbichler A, Kerschbaum J, Gopaluni S, et al. Trimethoprim-sulfamethoxazole prophylaxis prevents severe/life-threatening infections following rituximab in antineutrophil cytoplasm antibody-associated vasculitis. Ann Rheum Dis 2018;77:1440-7.
3 Fardet L, Petersen I, Nazareth I. Common infections in patients prescribed systemic glucocorticoids in primary care: a population-based cohort study. PLoS Med 2016;13:e1002024.

4 Yoshida K, Solomon DH, Kim SC. Active-comparator design and new-user design in observational studies. Nat Rev Rheumatol 2015;11:437-41.

5 Stone JH, Merkel PA, Spiera R, et al. Rituximab versus cyclophosphamide for ANCAassociated vasculitis. N Eng/ J Med 2010;363:221-32.

6 Stegeman CA, Cohen Tervaert JW, de Jong PE, et al. Trimethoprim-sulfamethoxazole (co-trimoxazole) for the prevention of relapses of Wegener's granulomatosis. $N$ Eng/ J Med 1996;335:16-20.

7 Park JW, Curtis JR, Moon J, et al. Prophylactic effect of trimethoprim-sulfamethoxazole for Pneumocystis pneumonia in patients with rheumatic diseases exposed to prolonged high-dose glucocorticoids. Ann Rheum Dis 2018;77:644-9.

8 Talan DA, Mower WR, Krishnadasan A, et al. Trimethoprim-sulfamethoxazole versus placebo for uncomplicated skin abscess. N Engl J Med 2016;374:823-32. 